\title{
Efficacy of electro-acupuncture for gastrointestinal motility after colorectal cancer surgery: study protocol for a randomized controlled trial
}

\section{yixuan Feng}

China Academy of Chinese Medical Sciences Institute of Acupuncture and Moxibustion

\section{Muwen Qu}

China Academy of Chinese Medical Sciences Guanganmen Hospital

\section{Xiumei Gao}

China Academy of Chinese Medical Sciences Xiyuan Hospital

yuru Zhang

Beijing rectum hospital

\section{Xiaoqiang Jia}

China Academy of Chinese Medical Sciences Xiyuan Hospital

\section{Yinqiu Gao}

China Academy of Chinese Medical Sciences Guanganmen Hospital

\section{Weiwei Cao}

China Academy of Chinese Medical Sciences Xiyuan Hospital

\section{Junyi Li}

China Academy of Chinese Medical Sciences Guanganmen Hospital

\section{Bin Huang}

Beijing rectum Hospital

Hong Zhao ( $\sim$ hongzhaojournal@163.com )

\section{Study protocol}

Keywords: Electro-acupuncture, Postoperative gastrointestinal dysfunction, Colorectal cancer surgery, Randomized controlled trial

Posted Date: January 24th, 2020

DOI: https://doi.org/10.21203/rs.2.21821/v1

License: (c) (i) This work is licensed under a Creative Commons Attribution 4.0 International License.

Read Full License 


\section{Abstract}

Background Gastrointestinal dysfunction is a common and major complication after colorectal cancer surgery that results in significant psychological disturbance and economic burden. Although some studies have shown the potential therapeutic effect of acupuncture on gastrointestinal motility recovery, there is still a lack of high-quality evidence.

Methods The trial is designed as a multicentre, parallel-group, randomized, single-blinded (outcome assessors), superiority, randomized trial. A total of 160 eligible patients will be randomly assigned to two groups: the electro-acupuncture (EA) group and the routine treatment (RT) group. Patients in the EA group will receive EA combined with routine treatment. EA will be administered for 30 minutes, once a day, starting 24 hours after surgery and lasting for 3 days. Patients in the RT group will receive routine intervention lasting until the patients are discharged from the hospital. The primary outcome will be the time to first flatus. The secondary outcomes will include the time to first defecation, visual analogue scale (VAS) scores for postoperative incision pain or abdominal pain, quality of recovery-40 (QOR-40) scores and the length of hospital stay. These outcomes will be evaluated at $24 \mathrm{~h}$ as well as on the $2 \mathrm{nd}$ and $3 \mathrm{rd}$ days after surgery. Any side effects of the treatment will be observed and recorded.

Discussion We expect that the study results will provide high-level evidence to determine the effects of EA on gastrointestinal motility recovery after colorectal cancer surgery.

\section{Background}

Colorectal cancer is the third most common of all cancers ${ }^{[1]}$. Surgical resection is the primary treatment for most non-metastatic types of colorectal cancer ${ }^{[2]}$. Postoperative gastrointestinal dysfunction (PGD) is a common and major complication after surgery with general anaesthesia ${ }^{[3]}$. The main symptoms of PGD include delayed flatus and defecation, abdominal distention, abdominal pain, nausea, vomiting and ileus $^{[4]}$. The prevalence of PGD is $8 \%-28 \%$ in all surgeries ${ }^{[5]}$. Research shows that the incidence of postoperative nausea and vomiting is $30 \%[6]$, while the incidence of postoperative ileus (POI) after abdominal surgery is $10-30 \%{ }^{[7]}$. If these symptoms are inappropriately treated, they will affect the operation effect and prolong the length of hospital stay, resulting in a significant economic load on health-care institutions ${ }^{[8]}$. Reliable data demonstrate that the prolongation of hospitalization in the United States due to ileus has been estimated to cost $\$ 1,500$ per patient or $\$ 750,000,000$ annually ${ }^{[9]}$.

The types of prevention for PGD include the use of minimally invasive surgery, the routine use of prophylactic nasogastric tubes (NGTs), the immediate resumption of eating and drinking after surgery, and the use of combined isosmotic mechanical bowel preparation with oral antibiotics and alvimopan if opioid-based analgesia is used. The treatment for PGD primarily includes placement of an NGT to relieve intractable nausea and vomiting with abdominal distension, opioid minimization, ambulation, rational fluid replacement maintaining euvolemia, electrolyte repletion, and gum chewing ${ }^{[10]}$. These managements, however, do not completely prevent PGD and do not always provide complete relief of 
symptoms. In addition, some gastrointestinal motility drugs often come with undesirable side effects. Therefore, an effective prevention and treatment modality with less adverse effects for PGD is urgently needed.

Acupuncture has been identified as an effective method for the recovery of gastro-intestinal function. In fact, the US National Institutes of Health published a consensus statement in 1998 declaring that acupuncture can be an effective treatment for postoperative nausea and vomiting ${ }^{[11]}$. A systematic review showed that electro-acupuncture might have positive effects on postoperative bowel motility recovery and pain control ${ }^{[12]}$. More recent clinical studies have shown that acupuncture promotes the recovery of gastrointestinal function after surgery ${ }^{[13-16 .]}$. However, due to lack of high-quality supporting evidence, the use of acupuncture is limited in clinical practice for treating PGD. Given the current situation, we want to provide high-quality evidence of acupuncture treatment for PGD though this RCT trial. We also want to provide another effective prevention and treatment method for PGD.

\section{Objective $\{7\}$}

This study aims to validate the efficacy of electro-acupuncture for gastrointestinal motility (GI) recovery and for the prevention of gastrointestinal dysfunction after colorectal tumour surgery. We hypothesize that the efficacy of electro-acupuncture combined with routine treatment for improvements in GI motility recovery will be better than that of routine treatment alone. The study was registered in the ISRCTN Registry (ISRCTN13146325).

\section{Methods}

\section{Study design $\{8\}\{13\}$}

The trial is a multicentre, parallel-group, randomized, single-blinded (outcome assessors), superiority, trial comparing (a) electro-acupuncture (EA) combined with routine treatment (RT) versus (b) routine treatment in a standard anaesthetic setting of programmed colorectal tumour operations. A total of 160 eligible patients will be recruited from one of three hospitals in Beijing, China. According to their group assignment, patients will be assigned to the treatment group or control group receiving electroacupuncture combined with routine treatment or routine treatment alone, respectively. This protocol has been registered at the ISRCTN registry, a primary clinical trial registry recognized by the World Health Organization (WHO) and the International Committee of Medical Journal Editors (ICMJE).

The study design flowchart is presented in Fig. 1, and the study design schedule is shown in Table 1. 
Figure 1 flow chart

Table1 study design schedule

Study period

\begin{tabular}{|c|c|c|c|c|c|c|c|c|c|}
\hline \multirow{2}{*}{ Timepoint } & \multirow{2}{*}{$\begin{array}{l}\text { Enrollment } \\
-t_{1}\end{array}$} & \multirow{2}{*}{$\begin{array}{l}\text { Allocation } \\
0\end{array}$} & \multicolumn{6}{|c|}{ Post-allocation } & \multirow{2}{*}{$\begin{array}{l}\text { Close-out } \\
t_{x}\end{array}$} \\
\hline & & & $t_{0}$ & $t_{1}$ & $t_{2}$ & $t_{3}$ & $\ldots$ & $t_{x}$ & \\
\hline \multicolumn{10}{|l|}{ Enrollment: } \\
\hline Eligibility screen & $x$ & & & & & & & & \\
\hline Informed consent & $x$ & & & & & & & & \\
\hline Allocation & & $x$ & & & & & & & \\
\hline \multicolumn{10}{|l|}{ Interventions: } \\
\hline EA combined with RT & & & & $X$ & $X$ & $X$ & & & \\
\hline Routine treatment & & & & $x$ & $x$ & $x$ & & & \\
\hline \multicolumn{10}{|l|}{ Assessments: } \\
\hline Demographic data & $\mathrm{x}$ & & & & & & & & \\
\hline Time to first flatus & & & $x$ & $x$ & $x$ & $x$ & $x$ & $x$ & \\
\hline Time of first defecation & & & $x$ & $x$ & $x$ & $x$ & $x$ & $x$ & \\
\hline VAS & & & & $x$ & $x$ & $x$ & & & \\
\hline Quality of recovery- 40 & & & & $x$ & & $x$ & & & $x$ \\
\hline Hospital stays & & & & & & & & & $x$ \\
\hline AEs & & & & $x$ & $x$ & $x$ & $x$ & $x$ & $x$ \\
\hline
\end{tabular}

$-t_{1}$, time before operation; $t_{0}$, operation day; $t_{1}-t_{x}$, postoperative day 1 to postoperative day $X(=$ actual discharge day); 


\section{Study population}

\section{Trial location $\{5 \mathrm{~d}\}\{9\}$}

We will recruit participants from the in-patients of three hospitals: Guang'an Men Hospital, China Academy of Chinese Medical Sciences (CACMS); Xiyuan Hospital, CACMS; and Beijing rectum hospital. These three medical facilities are all tertiary hospitals and are all situated in Beijing, the capital of China.

\section{Inclusion criteria $\{10\}$}

Participants will be eligible if they meet all of the following criteria: (1) males or females who are scheduled for colorectal cancer surgery and are aged between 30 and 80 years old; (2) colon and rectal tumours have been diagnosed, and the tumour node metastasis (TNM) stage is less than stage 3; (3) the level of American Society of Anaesthesiologists (ASA) is I or II; (4) the score of the nutritional risk screening (NRS) 2002 is $\leq 3$; and (6) signed informed consent has been provided.

\section{Exclusion criteria $\{10\}$}

Participants will be excluded if they have any of the following conditions: (1) if they have been treated with acupuncture or opioid analgesics in the last month; (2) if they have a history of abdominal surgery in the last three months; (3) if they have mental disorders or have a history of taking sedatives and antidepressants for at least one year; (4) if they cannot be treated or do not accept electro-acupuncture treatment; and (5) if they have current severe cardiovascular, kidney or metabolic diseases.

\section{Study procedures}

\section{Recruitment $\{15\}\{26 a\}$}

The clinical recruitment staff will be in charge of the enrolment process. To achieve the target enrolment of participants, clinical recruitment flyers will be placed in the three hospitals. Participants will be screened based on the inclusion and exclusion criteria and will be informed of the purpose, procedures, treatments and possible risks of the trial as well as their right to discontinue participation in this study. The participants will be required to sign the informed consent forms before the trial. The recruitment staff is responsible for the storage of all the informed consent forms. Once participants sign the consent forms, a staff in charge of randomization process will then assign the recruited patients to different groups according to random numbers. .

\section{Randomization $\{16 \mathrm{a}\}$ \{16b\} $\{16 \mathrm{c}\}$}

The central random method will be used. We will generate random numbers with Excel software $\square$ Microsoft Corporation $\varangle$ Washington, the United States of America $\llbracket$. There will be a staff member who will keep the random numbers. When recruiters identify a subject who meets the criteria for participation, they will obtain a sequence number for that subject from the researcher in charge of the randomization 
numbers via WeChat or telephone from the staff. The eligible participants will be randomly assigned to the EA group or the RT group according to the random numbers.

\section{Blinding $\{17 \mathrm{a}\}\{17 \mathrm{~b}\}$}

Because the EA group and RT group will have different characteristics, it is not feasible to blind both the operators and patients. Instead, we will adopt a single-blinded trial design in which both the outcome evaluator and statistical analyst will be blinded to the groupings and will not be involved in any part of the treatments during the trial to ensure that there is no form of bias in terms of the results. At the end of the observation period, un-blinding will be permissible only after database blinding approval and data blocking.

\section{Participating trial physicians $\{10\}$}

The participating trial physicians and acupuncturists will be employees of the three hospitals. Surgeons and anaesthetists with at least 10 years of clinical experience will be responsible for the colorectal cancer surgery and anaesthetic procedures. A licensed acupuncturist, preferably with a Master's or Doctorate degree in acupuncture and at least 2 years of acupuncture experience, will be responsible for delivering the electro-acupuncture treatment.

\section{Interventions $\{11 \mathrm{a}\}$}

\section{Surgery and anaesthetic procedure}

The patients will undergo either laparotomy or laparoscopy in one of the three hospitals. A standardized anaesthetic protocol that includes continuous epidural anaesthesia combined with general anaesthesia will be applied to all eligible patients.

\section{Routine treatment}

All participants will undergo routine treatment, which will include routine intravenous nutrition lasting until the patient is discharged from the hospital. When patients have incisional pain/abdominal pain, an analgesic pump will be used lasting for 3 days after surgery. According to the degree of pain, and if the VAS score is greater than 6 points, the patients will be able to implement self-controlled pain relief via a button on the pump. Flurbiprofen axetil injection will be administered intravenously at a dose of $50 \mathrm{mg}$ at times when the pain is unbearable 30 minutes after self-control of the analgesic pump has ended.

\section{Electro-acupuncture treatment}

Participants in the EA group will receive electro-acupuncture. 
The acupoints will be the following, as they are known to promote gastrointestinal motility: bilateral ST36 (Zusanli), bilateral ST37 (Shangjuxu), bilateral LI4 (Hegu), and bilateral LR3 (Tai chong); these acupoints will be located according to the WHO International Standard Acupuncture Points ${ }^{[17]} \otimes$ Fig. $2 \rrbracket$.

Figure 2 acupionts

Stainless-steel needles and an SDZ-II electro-acupuncture device will be used in this trial. The acupuncture needles are disposable and sterile and measure $0.25 \times 25 \mathrm{~mm}$ and $0.25 \times 40 \mathrm{~mm}$ in size (Suzhou Medicine Equipment Co., Ltd., Suzhou, China.). After standard disinfection of the acupoints with $75 \%$ ethanol solution, the acupuncturist will position the disposable medical needles perpendicular to the skin at the subject's acupoints and then insert them into the skin. The needles will be placed into the skin at a depth of $2 \mathrm{~cm}$ at ST36, ST37, and LI4 and at a depth of $1 \mathrm{~cm}$ at LR3. Each needle will be rotated until the patient feels pain, numbness, distension or a heavy sensation in the local area. The Hwato SDZ-II electro-acupuncture device (Suzhou Medical Appliance Factory, Suzhou, China) will then be connected to two pairs of needles: bilateral ST36 (Zusanli) and bilateral LR3 (taichong); then, the density wave will be selected. The magnitude and intensity of the electro-acupuncture current will be determined by the patient's tolerance. Electro-acupuncture will be administered for 30 minutes, once a day, starting 24 hours after surgery and lasting for 3 days.

Patients allocated to the EA group will receive routine treatment with electro-acupuncture. Patients allocated to the RT group will receive the same routine treatment without electro-acupuncture.

\section{Concomitant care and intervention $\{11 \mathrm{~d}\}$}

During the treatment and follow-up period, the participants will be advised to avoid other drugs and therapies for gastrointestinal dysfunction, such as Chinese patent drugs and Western medicines. For any unallowed treatment that has already been used, relevant information will be recorded in detail in the patient's Case Report Form (CRF).

For complicated chronic diseases, patients must continue taking their routine medications and other therapies. The research staff will record the names of those diseases, medications and therapies in the CRF.

\section{Discontinuation of the intervention $\{11 \mathrm{~b}\}$}

The intervention will be terminated when severe adverse events occur. These severe adverse events include fainting, needle breakage and haematoma. If these events occur, the intervention will be stopped, and the patients will receive appropriate treatment in a timely manner.

The intervention will also be terminated when patient have serious postoperative infections or other major adverse reactions caused by the operation.

Outcome measures $\{12\}$ 
Similar outcomes have been applied in previous studies on gastrointestinal dysfunction, such as the time to the first passage of flatus, the time of the first defecation, visual analogue scale (VAS), quality of life ${ }^{[4,18]}$, and the length of hospital stay ${ }^{[19-21]}$. Thus, we will adopt these outcomes in this study due to their wide application.

\section{Primary outcome}

The primary outcome is the time to the first flatus, which will be recorded by the subject and nurses. The time to the first passage of flatus refers to the interval between the moment of the first passage of flatus and the moment when the subject returns to the ward after surgery.

\section{Secondary outcomes}

1. The time of first defecation: The participants and nurses will be asked to record the time of first defecation. The time of first defecation refers to the interval between the moment of the first defecation and the moment when the subject returns to the ward safely after surgery.

2. VAS scale of postoperative incision pain or abdominal pain: The degree of pain will be assessed with the VAS scale ${ }^{[22]}$. The VAS score will be recorded at $24 \mathrm{~h}$ as well as on the $2 \mathrm{nd}$ day and 3 rd day after surgery.

3. Quality of recovery-40 (QoR-40): The QoR-40 is a 40-item recovery-specific and patient-rated questionnaire measuring five aspects: physical comfort (12 items), emotional state (nine items), physical independence (five items), psychological support (seven items) and pain (seven items) ${ }^{[23]}$. The total score of the QoR-40 is 200 . Higher scores indicate better health status. The QoR-40 will be conducted at $24 \mathrm{~h}$ and on the 3rd day after surgery.

4. Length of hospital stay: Period of time between when the participant is hospitalized and when the participant is discharged from the hospital.

\section{Data collection and management $\{5 d\}\{18 a\}\{19\}$}

The evaluator will be responsible for the collection of baseline characteristic data. The baseline variables are as follows: age, sex, nationality, marital status, height, weight, operation mode, operation duration, intraoperative blood loss, intraoperative infusion volume, medical history, treatment history, relevant examinations, anaesthesia type, and Constipation Scoring System (CSS) and QoR-40 scores.

We will consolidate the baseline variables, outcome measures, drug consumption, adverse events, and safety evaluations into a single CRF. The evaluator will complete the CRF accurately and promptly at every observation time.

Data monitoring and management will be performed by the institute of acupuncture and moxibustion, CACMS, every three months. Two assistants will enter all data into an electronic database via double data entry. The statistical manager will be responsible for source data organizing, coding, range checking of data values and converting data to ensure data quality. 
The research documents will be preserved for at least five years after publication. If reviewers or readers have any questions concerning our published data, they can contact the corresponding author for access to the original data. The private information of patients will be anonymous to ensure participant confidentiality.

\section{Statistical analysis}

\section{Sample size $\{14\}$}

The mean change in the time to first flatus will be used as the indicator for the efficacy evaluation in the calculation of the sample size. The results from a previous study ${ }^{[24]}$ show that the mean time to first flatus is $96 \pm 38.4$ hours with routine treatment. According to our previous unpublished pilot study results, the time of first flatus with electro-acupuncture treatment might be $75 \pm 46.5$ hours. We performed an inferiority test to calculate the appropriate trial sample size with $90 \%$ power, an alpha of 0.05 , and a $20 \%$ drop-out rate. Thus, we plan to include a total of 160 patients.

\section{Statistical methods $\{20$ a $\}$}

The statistical analysis will be performed by a blinded statistician using SPSS 17.0 software (SPSS Inc., Chicago, IL, USA) in the Clinical Evaluation Center of CACMS. Demographic and clinical data will be analysed at baseline to measure the balance between the two groups. The intention-to-treat principal will be used in the data analysis. Statistical treatments will be two-sided tests, and a P-value $<0.05$ will be considered statistically significant. Descriptive statistics will be used to compare the baseline measures and patients' characteristics between the two groups. We will perform baseline adjusted analyses if the baseline data are unbalanced.

Continuous data will be expressed as the mean \pm standard deviation and median, maximum and minimum values, while categorical data will be described by frequencies and percentages.

Analysis methods will be selected according to the data distribution characteristics: For comparison between the two groups, one-way ANOVA (normal distribution) or non-parametric test (non-normal distribution) will be applied for measurement data. The counting data will be analysed by one-way ANOVA. For the comparison of baseline data, paired t tests or non-parametric tests will be used for measurement data, and rank sum tests will be used for counting data.

Linear regression analysis will be used to evaluate the factors affecting the curative effect.

\section{Sub-analyses $\{20 \mathrm{~b}\}$}

Sub-analyses will be performed on the operation methods: open surgery and laparoscopic surgery.

\section{Missing data and sensitivity analysis $\{20 \mathrm{c}\}$}


We will record the time and reason for the missing data and will use the multiple imputation adjustment approach to analyse those missing data. We will also perform a sensitivity analysis for the various datasets after the main analysis to enable us to judge the impact of missing data on the results.

\section{Quality control $\{5 d\}\{11 c\}\{18 b\}\{21 a\}\{21 b\}\{22\}\{23\}$}

This protocol was developed after consulting acupuncture proctologists, anaesthetists, statisticians, and methodologists. All staff will receive training in advance in terms of the trial protocol, participant selection, electro-acupuncture intervention, effect assessment and methods for filling out the CRF. The staff will be examined after training to ensure the consistency of electro-acupuncture treatment and the evaluation method.

Patients will be informed of the importance and significance of this project in advance. During the whole treatment process, there will be a research staff member who will observe the patient's situation and answer questions for the patient at any time to ensure good patient compliance. For participants who discontinue or deviate from intervention protocols, we will record the causes and clinical outcomes as much as possible.

During each visit, uninduced questions were used to detect adverse events from patients. Adverse events may also be voluntarily reported by the patient during the visit or detected by physical examination, laboratory examination, or other means. The outcome evaluator will record the details of the adverse events on the CRF, including the name of the adverse events, date of occurrence and end, severity, relationship to the intervention, and impact on the intervention for conversion.

A special research team from the institute of acupuncture and moxibustion, China academy of Chines medical sciences, which is independent from the investigators and sponsor, will perform external monitoring of this study in the three hospitals every 3 months during the study to ensure the authenticity of the data. An advisory board will follow the trial and give advice when necessary. The data monitoring committee (DMC) has been established independent of the sponsor, and there is no conflict of interest. The DMC is responsible for monitoring the trial progression and guaranteeing patient safety. We have not specified interim analyses and stopping guidelines of the trial. These will be supplied if the DMC requests interim analyses.

\section{Ethics and dissemination}

\section{Ethics $\{27\}\{29\}\{30\}$}

This study was designed in accordance with Chinese corresponding regulations and the principles of the 1996 Declaration of Helsinki. It has been approved by the Ethics Committee of the Institute of 
Acupuncture and Moxibustion of CACMS (2017-12-25-1), Beijing rectum hospital (2019ELLHA-002-01), Guang'anmen Hospital, CACMS (2018-006-KY-01) and Xiyuan Hospital, CACMS (2018XLA010-1).

Case Report Forms (CRFs) will be created based on the study protocol. Data will be entered in the CRF within $24 \mathrm{~h}$ of collection by blinded assessors and will be securely stored at the study centre until completion of the trial. Additionally, the participant data obtained in this research will not be used for other purposes, and participants' personal information will be kept confidential. The sponsor, statistical manager, and monitoring manager will have access to the final trial dataset and disclosure of contractual agreements.

After the trial, we will provide a certain amount of economic compensation or free treatment for those who suffer harm from trial participation.

\section{Dissemination $\{31 \mathrm{a}\} 31 \mathrm{~b}\}$}

Publications will be planned in English versions. Regardless of the findings, we plan to disseminate all of the trial results in conferences or publications.

This protocol was written following the Recommendations for Interventional trials (SPIRIT) checklist (see Additional file 1). The future report will follow the Consolidated Standards of Reporting Trials (CONSORT) guidelines $^{[25]}$ and revised standards for reporting interventions in Clinical Trials of Acupuncture ${ }^{[26]}$. We are not intended to use professional writers.

\section{Discussion}

The objective of this study is to validate the efficacy of electro-acupuncture for gastrointestinal motility recovery after colorectal cancer surgery.

In fact, acupuncture has been used to treat various gastrointestinal disorders in China for thousands of years, and acupuncture has been proven to have good clinical efficacy. To prevent PGD by EA, four acupoints, ST36, ST37, LI4, and LR3, were selected for this trial. ST36 and ST37 are located on the lower legs, and both belong to the Stomach Meridian of Foot-Yangming, which is closely related to gastrointestinal function. Based on traditional Chinese medicine theory, LI4 and LR3 promote qi and blood circulation as well as the recovery of body functions after surgery. Some studies have focused on the effect of acupuncture at these four acupoints on the recovery of gastrointestinal function. Some RCTs [20,27-28] have shown that EA on these acupoints can shorten the time of first flatus and defecation and can reduce the length of hospital stay. However, the number of cases in these studies is small, and the quality of research is not high. Experimental studies ${ }^{[29-30]}$ have shown that acupuncture performed at acupoints on the stomach meridian of foot-yangming has a regulatory effect on gastrointestinal electrophysiology, the secretion of gastric and intestinal fluids, gastrointestinal hormones and blood flow. These factors might be involved in the possible mechanism by which acupuncture of these acupoints can promote gastric motility. Therefore, we chose these four acupoints to treat PGD. Although some 
studies show that acupoints located in the abdomen also have great efficacy in the recovery of gastrointestinal dysfunction, we did not select them because the abdomen is covered by surgical dressings after surgery; thus, it is not convenient to manipulate acupuncture in this area.

For this study, we chose routine treatment as a control treatment instead of placebo acupuncture treatment. The first reason for this decision is that the outcomes of gastric motility recovery are relatively objective, and the placebo effect has little effect on results. In addition, a systematic review suggested that acupuncture has a better effect than sham acupuncture for promoting the recovery of gastrointestinal function ${ }^{[31]}$. The purpose of this study is to verify whether routine treatment combined with EA is superior to routine treatment alone for gastrointestinal motility recovery after colorectal cancer surgery; thus, we chose routine treatment as the control group. In order to blind the patients, we will arrange participants in distant beds and draw the curtains during treatment periods to ensure that the patients do not know the group assignment.

The recovery of postoperative $\mathrm{GI}$ function is multifactorial and affects other indicators, such as hospital stay ${ }^{[32-33]}$. Therefore, in addition to measuring gastrointestinal function, we also selected other related outcomes, including incision or abdomen pain, postoperative quality of life and length of hospital stay, to evaluate the overall efficacy of EA on patients after colorectal surgery.

In the guideline of the management of colorectal cancer, enhanced recovery after surgery (ERAS) programmes are recommended for the perioperative management of colorectal surgery ${ }^{[2]}$. One of the programmes involves the prevention of postoperative ileus. We expect that this study will provide highquality evidence for EA combined with routine treatment to promote GI motility recovery and provide scientific evidence for the application of EA in ERAS programmes.

\section{Declarations}

\section{Ethical approval and consent to participate}

The names of the ethical review committees that have reviewed and approved this study are as follows:

1. Ethics Committee of Institute of Acupuncture and Moxibustion of CACMS. Reference number 2017-12$25-1$.

2. Ethics Committee of Guang'anmen Hospital, CACMS. Reference number 2018-006-KY-01 2015 EC043

3. Ethics Committee of Xiyuan Hospital『CACMS. Reference number 2018XLA010-1

4. Ethics Committee of Beijing rectum hospital. Reference number 2019ELLHA-002-01.

Written informed consent will be obtained from each patient prior to enrolment.

\section{Consent for publication}

Not Applicable 
Availability of data and materials

The datasets generated and/or analysed during the current study are available from the corresponding author upon reasonable request.

\section{Competing interests $\{28\}$}

The authors declare that they have no competing interests.

\section{Funding $\{4\}\{5 b\{5 c\}$}

This trial is funded by grants from the China Academy of Chinese Medical Sciences (ZZ11-047). The funder had no role in the design of the study and collection, analysis, and interpretation of data, and in writing the manuscript.

The trial sponsor is professor Xianghong Jing, her email address is jxhtjb@263.net. The sponsor had no role in study design $\llbracket$ collection, analysis, interpretation of data and in writing the protocol.

\section{Acknowledgements}

We acknowledge Guang'anmen Hospital, Xiyuan Hospital, CACMS and Beijing rectum hospital for their cooperation in the recruitment and treatment of patients and the ethics reviews. We would like to express our gratitude to the participants in this trial for their cooperation.

\section{Abbreviations}

PGD, postoperative gastrointestinal dysfunction; POI, postoperative ileus; RCT, randomized controlled trial; $\mathrm{GI}$, gastrointestinal motility; EA, electro-acupuncture; RT, routine treatment; WHO, World Health Organization; ICMJE, International Committee of Medical Journal Editors; CACMS, China Academy of Chinese Medical Sciences; CRF, Case Report Form; VAS, visual analogue scale; QoR-40, quality of recovery score-40; CSS, constipation scoring system; DMC, data monitoring committee.

\section{Authors' contributions\{5a\}}

$\mathrm{HZ}$ was responsible for the study design and development of the protocol. YXF drafted the protocol manuscript and participated in study coordination. MWQ, XMG, and YRZ participated in the revision of the manuscript and provided administrative, technical, or material support. XQJ, YQG, WWC, JYL and BH participated in participant recruitment and data collection. All authors read and approved the final manuscript.

\section{Trial status $\{3\}$}

This trial is currently recruiting participants. This is the protocol of the second version (v2.0 dated

29 December 2017). All protocol versions have been submitted to the ethics committees of the three hospitals. Research ethics committee review board approvals are kept with the ethics committees of the 
three hospitals. The date of enrolment of the first participant to the trial is 20 April 2018. The recruitment will be completed by 31 October 2020 .

\section{Author details $\{5 a\}$}

${ }^{1}$ Institute of Acupuncture and Moxibustion, China Academy of Chinese Medical Sciences, Beijing 100700, China. ${ }^{2}$ Guang'anmen Hospital, China Academy of Chinese Medical Sciences, Beijing 100053, China. ${ }^{3}$ Xiyuan Hospital, China Academy of Chinese Medical Sciences, Beijing 100053, China. 4 Beijing rectum hospital, Beijing 100120, China.

\section{References}

[1]. Ferlay J, Shin H.R, Bray F, Forman, D ,Parkin, D.M. Cancer incidence and mortality worldwide: IARC CancerBase No. International Agency for Research on Cancer.2013; 2.

[2]. Brown K.G.M, Solomon M.J, Mahon K ,O Shannassy S. Management of colorectal cancer. BMJ.2019; 366: 14561

[3]. Steinbrook, Richard A. Epidural Anesthesia and Gastrointestinal Motility. Anesthesia \& Analgesia.1998; 86(4): 837-844.

[4]. Tan Y., Zhao Y., He T., Ma Y., Cai W. ,Wang Y. Efficacy and safety of auricular point acupressure treatment for gastrointestinal dysfunction after laparoscopic cholecystectomy: study protocol for a randomized controlled trial. Trials.2016; 17(1): 280

[5].Gu K.M, Zeng M, Liu M, Mu C.X. Comparison of several treatment methods for postoperative abdominal distention (article in Chinese). Harbin Medical Journal. 2007;02:22-24

[6]. Mihara T, Tojo K, Uchimoto K, Morita S ,Goto T. Reevaluation of the Effectiveness of Ramosetron for Preventing Postoperative Nausea and Vomiting. Anesthesia \& Analgesia.2013; 117(2): 329-339.

[7].Venara A, Neunlist M, Slim K, Barbieux J, Colas P.A , Hamy A, et al. Postoperative ileus: Pathophysiology, incidence, and prevention. J Visc Surg.2016; 153.

[8]. Vather, R. ,Bissett, I. Management of prolonged post-operative ileus: Evidence-based recommendations. Anz J Surg.2013; 83(5): 319-324.

[9]. Livingston, E.H. ,Passaro, E.P. Postoperative ileus. Digest Dis Sci.1990; 35(1): 121-132.

[10]. Hedrick T.L, McEvoy M.D, Mythen M.M.G, Bergamaschi R, Gupta R , Holubar S.D, et al. American Society for Enhanced Recovery and Perioperative Quality Initiative Joint Consensus Statement on 
Postoperative Gastrointestinal Dysfunction Within an Enhanced Recovery Pathway for Elective Colorectal Surgery. Anesthesia \& Analgesia.2017: 1.

[11]. Morey, Sharon S. NIH issues consensus statement on acupuncture. Am Fam Physician.1998; 57(10): 2545-6.

[12]. Garcia MK, M.J.H.R. Systematic review of acupuncture in cancer care: a synthesis of the evidence. J Clin Oncol.2013; 31(7): 952-960.

[13]. Simon Ng, Wing L, Mak, T, Sophie H, Jimmy L, Cherry W, et al. Electroacupuncture Reduces Duration of Postoperative lleus After Laparoscopic Surgery for Colorectal Cancer. Gastroenterology.2012; 144(2): 307-313.e1.

[14]. Zhang Z., Wang C., Li Q., Zhang M., Zhao H. ,Dong L., et al. Electroacupuncture at ST36 accelerates the recovery of gastrointestinal motility after colorectal surgery: a randomised controlled trial. Acupunct Med.2014; 32(3): 223-6.

[15]. Qiu W. ,Jiang J.. Effect of acupoint injection of Neostigmine on gastrointestinal function after cholecystectomy. Journal of Acupuncture and Tuina Science.2015; 13(6): 368-372.

[16]. Lu Z, Dong H, Wang Q, Xiong L ,Hardman J.G. Perioperative acupuncture modulation: more than anaesthesia. Brit J Anaesth.2015; 115(2): 183-193.

[17]. WHO Regional Office for the Western Pacific .WHO standard acupuncture point locations in the Western Pacific Region. WorldHealthOrganization.2008.

[18]. Zhao W., Li J., Wang Y., Liu J., Chen Y. ,Zhao G., et al. Efficacy and safety of the "Xingnao Kaiqiao" acupuncture technique via intradermal needling to treat postoperative gastrointestinal dysfunction of laparoscopic surgery: study protocol for a randomized controlled trial. Trials.2017; 18(1): 567.

[19]. Kim G. Electroacupuncture for postoperative pain and gastrointestinal motility after laparoscopic appendectomy (AcuLap): study protocol for a randomized controlled trial. Trials.2015; 16(1): 461.

[20]. Liu M., Wang C., Wu Z. ,Li N. Electroacupuncture for the prevention of postoperative gastrointestinal dysfunction in participants undergoing vascular laparotomy under general anesthesia: a randomized controlled trial. Chin Med-Uk.2017; 12(1): 5.

[21]. Kim K.H, Kim D.H, Bae J.M, Son G.M, Kim K.H ,Hong S.P, et al. Acupuncture and PC6 stimulation for the prevention of postoperative nausea and vomiting in patients undergoing elective laparoscopic resection of colorectal cancer: a study protocol for a three-arm randomised pilot trial. Bmj Open.2017; 7(1): e013457

[22]. Paula K, J. W.P, Alan T ,André M. Is the Pain Visual Analogue Scale Linear and Responsive to Change? An Exploration Using Rasch Analysis. Plos One.2014; 9(6): e99485-. 
[23]. Myles P.S, Weitkamp B, Jones K, Melick J , Hensen S..Validity and reliability of a postoperative quality of recovery score: the QoR-40. Brit J Anaesth.2000; 84(1): 11-15.

[24]. Zingg U, Miskovic D, Pasternak I, Meyer P, Hamel C.T ,Metzger U. Effect of bisacodyl on postoperative bowel motility in elective colorectal surgery: a prospective, randomized trial. Int $\mathrm{J}$ Colorectal Dis.2008; 23(12): 1175

[25]Martins J, Sousa LM, Oliveira AS. Recomendações do enunciado CONSORT para o relato de estudos clínicos controlados e randomizados. Medicina. 2009;42(1):9-21.

[26]Pherson HM, Altman DG, Hammerschlag R, Li Y, Taixiang W, White A, et al. Revised Standards for Reporting Interventions in Clinical Trials of Acupuncture (STRICTA): extending the CONSORT Statement. PLoS Med. 2010;8(6):7.

[27]. Pan H, Li J, Zhao Y ,Li N. A Randomized Controlled Clinical Trial for Electroacupuncture Treatment of Post-surgical Gastrointestinal Dysfunction in Patients Undergoing Laparotomy. Zhen ci yan jiu.2016; 41(5): 457-461.

[28]. Liu M.Y, Wang C.W,Wen Q, Li N. Clinical observation of gastrointestinal dysfunction after vascular surgery prevented by electroacupuncture. Chinese Acupuncture \& Moxibustion.2016.

[29]. Yang Q, Xie Y, Zhang M, Huang B, Zhang C ,Li H, et al. Effect of electroacupuncture stimulation at Zusanli acupoint (ST36) on gastric motility: possible through PKC and MAPK signal transduction pathways. Bmc Complem Altern M.2014; 14(1): 137.

[30]Liu H, Zhao H, Yi S.X. Study on the effect of acupuncture Foot Yangming Stomach Meridian on gastrointestinal function in recent 10 years. Chinese Journal of Information on Traditional Chinese Medicine.1999(12): 19-21.

[31]. Liu Y, H. M.B, Lin Z.A, Xinfeng G, Chuanjian L, Changli X.., et al. Acupuncture and Related Therapies for Treatment of Postoperative Ileus in Colorectal Cancer: A Systematic Review and MetaAnalysis of Randomized Controlled Trials. Evid-Based Compl Alt.2018; 2018: 1-18.

[32]. Fiore J.F, Bialocerkowski A, Browning L, Faragher I.G , Denehy L. Criteria to Determine Readiness for Hospital Discharge Following Colorectal Surgery: An International Consensus Using the Delphi Technique. Dis Colon Rectum.2012; 55(4): 416

[33]. Mythen $\square$ Michael G. Postoperative Gastrointestinal Tract Dysfunction. Anesthesia \& Analgesia.2005; 100(1): 196-204.

\section{Figures}




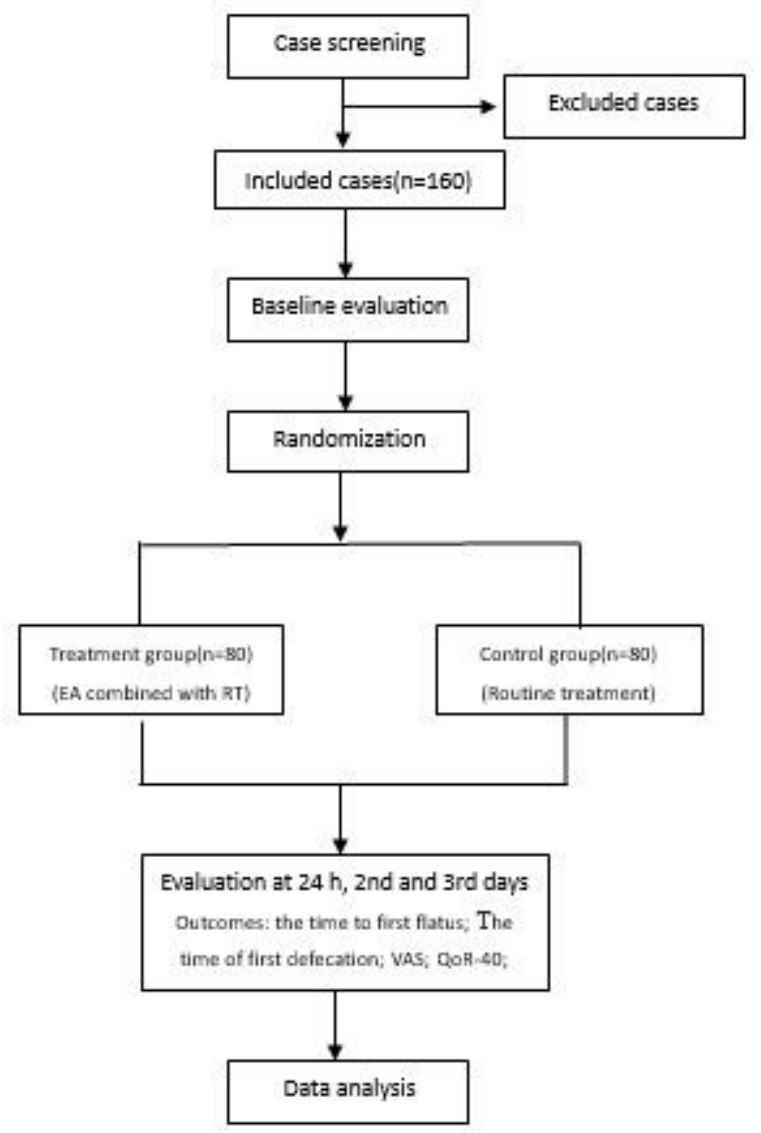

Figure 1 flow chart

Figure 1

flow chart 


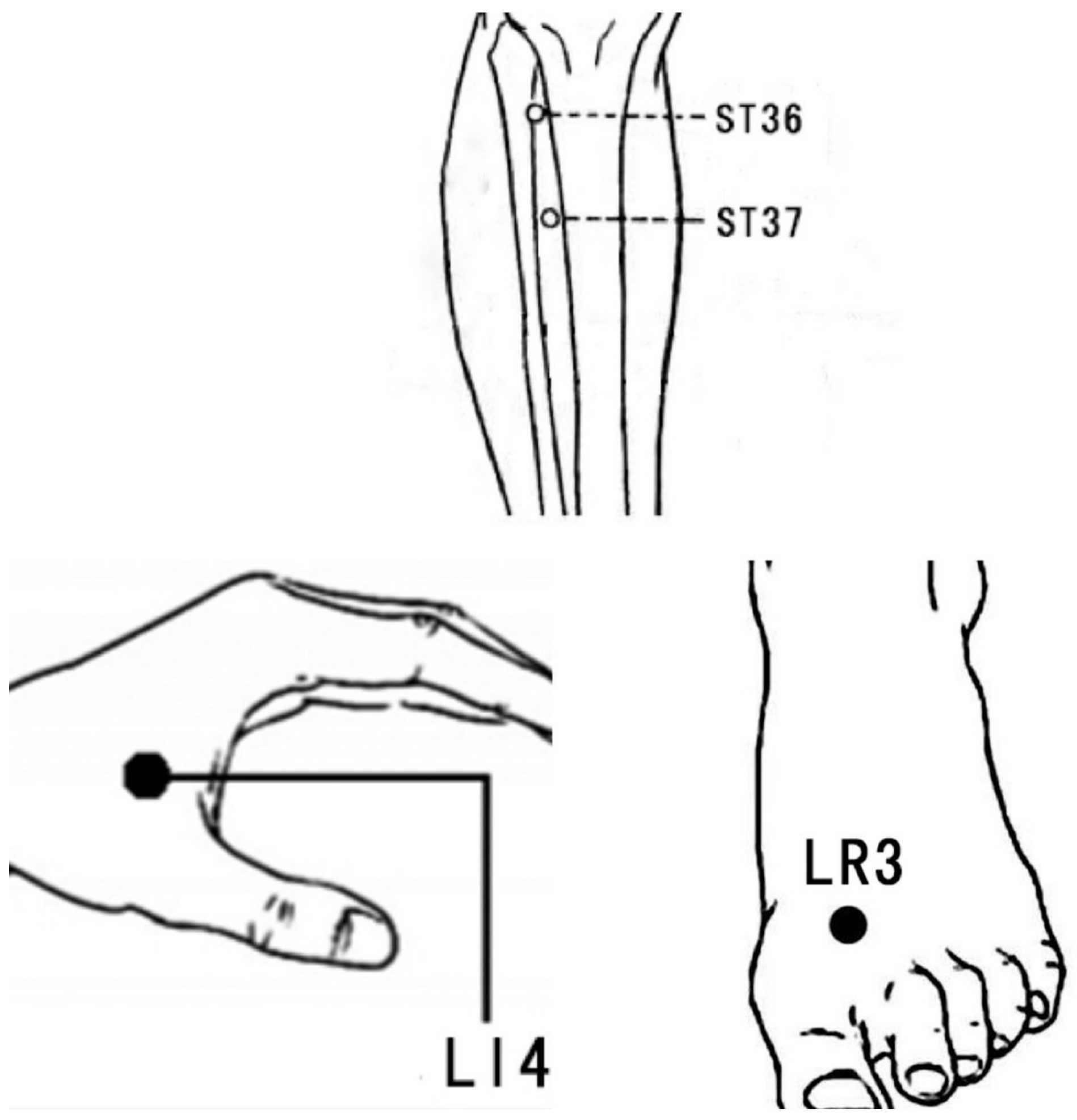

Figure 2

acupionts

Supplementary Files

This is a list of supplementary files associated with this preprint. Click to download. 
- Beijingrectumhospital.pdf

- additinalfile1SPIRITchecklist.doc

- FundingDocumentation.jpg

- XiyuanHospital.pdf

- InstituteofAcupunctureandMoxibustion.PDF 